\title{
Global maps of lunar neutron fluxes from the LEND instrument
}

1. M. L. Litvak ${ }^{1, *}$,

2. I. G. Mitrofanov ${ }^{1}$,

3. A. Sanin ${ }^{1}$,

4. A. Malakhov ${ }^{1}$,

5. W. V. Boynton ${ }^{2}$,

6. G. $\mathrm{Chin}^{3}$,

7. G. Droege ${ }^{2}$,

8. L. G. Evans ${ }^{4}$,

9. J. Garvin ${ }^{3}$,

10. D. V. Golovin ${ }^{1}$,

11. K. Harshman ${ }^{2}$,

12. T. P. McClanahan,

13. M. I. Mokrousov ${ }^{1}$,

14. E. Mazarico,

15. G. Milikh

16. G. Neumann ${ }^{3}$,

17. R. Sagdeev ${ }^{5}$,

18. D. E. Smith ${ }^{3}$,

19. R. Starr 6

20. M. T. Zuber ${ }^{7}$

Article first published online: 5 JUN 2012

DOI: 10.1029/2011JE003949

Copyright 2012 by the American Geophysical Union

Issue

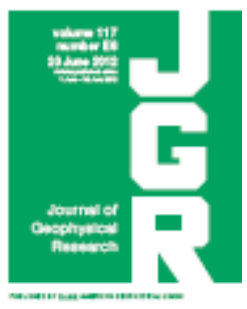

Journal of Geophysical Research: Planets (1991-2012)

Volume 117, Issue E12, December 2012 
Additional Information(Show All)

$\underline{\text { How to CiteAuthor InformationPublication History }}$

\section{SEARCH}

Search Scope

Search String

- $\quad$ AGU EASI Search $>$

- $\quad$ Browse by Index Term $>$

\section{ARTICLE TOOLS}

- Get PDF (7481K)

- $\quad$ Save to My Profile

- E-mail Link to this Article

- Export Citation for this Article

- $\quad$ Get Citation Alerts

- $\quad$ Request Permissions

More Sharing ServicesShare|Share on citeulikeShare on connoteaShare on delicious Share on www.mendeley.comShare on twitter

- $\underline{\text { Abstract }}$

- Article

- References

- Supporting Information

- $\quad$ Cited By

Get PDF (7481K)

- $\quad$ Go here for SFX

\section{Keywords:}

- LEND;

- Moon;

- epithermal neutrons;

- hydrogen;

- water 


\begin{abstract}
[1] The latest neutron spectrometer measurements with the Lunar Exploration Neutron Detector (LEND) onboard the Lunar Reconnaissance Orbiter (LRO) are presented. It covers more than 1 year of mapping phase starting on 15 September 2009. In our analyses we have created global maps showing regional variations in the flux of thermal (energy range $<0.015 \mathrm{eV}$ ) and fast neutrons $(>0.5 \mathrm{MeV})$, and compared these fluxes to variances in soil elemental composition, and with previous results obtained by the Lunar Prospector Neutron Spectrometer (LPNS). We also processed data from LEND collimated detectors and derived a value for the collimated signal of epithermal neutrons based on the comparative analysis with the LEND omnidirectional detectors. Finally, we have compared our final (after the data reduction) global epithermal neutron map with LPNS data.
\end{abstract}

\title{
1. Introduction
}

[2] The Moon is continuously bombarded by high energy charged particles (primarily protons and alpha particles) from Galactic Cosmic Rays (GCR). The result of such interactions is production of neutrons over a wide energy range within the upper $(\sim 1 \mathrm{~m})$ subsurface. The observation of the neutron leakage spectrum may provide information about the hydrogen content of the Moon itself, as well as about the space environment (for example variations of GCRs, see Mitrofanov et al. [2009]).

[3] Regional variations of neutron flux in the thermal energy range (neutrons with energies below $0.4 \mathrm{eV}$ ) correlate with concentrations of major and minor lunar soil forming elements (like $\mathrm{Fe}, \mathrm{Ti}, \mathrm{Gd}, \mathrm{Sm}$ ) having large macroscopic absorption cross sections. For example, the distribution of Fe and Ti is highly "non-homogeneous" between the nearside mare basins and farside highlands and as a result produces the significant regional variations of thermal neutrons across the lunar surface [see, e.g.,Lawrence et al., 2002; Elphic et al., 1998, 2000, 2002]. The epithermal energy range (neutrons with energies from $0.4 \mathrm{eV}$ up to $100 \mathrm{keV}$ ) is another key subsurface composition marker because the intensity of neutron flux in this energy range is sensitive to the abundance of hydrogen. Even a small amount $(\sim 100 \mathrm{ppm})$ of H causes significant depression of epithermal neutron flux. Finally, fast neutrons (neutrons with energies from $100 \mathrm{keV}$ up to $15 \mathrm{MeV}$ ) may be used to analyze the average composition of lunar soil via correlation of intensity of fast neutron flux and average atomic mass of the lunar soil.

[4] More than 10 years ago lunar neutron leakage spectra were acquired by the Neutron Spectrometer instrument onboard the Lunar Prospector mission (LPNS; for instrument details, see Feldman et al. [2004]). LPNS was able to create global maps of lunar neutron albedo in different energy ranges including observations of thermal, epithermal and fast neutrons [ Feldman et al., 1998a, 1998b; Maurice et al., 2004]. The most intriguing result was the observation of regional variations of epithermal neutron flux across the lunar surface. LPNS revealed vast polar regions of epithermal neutron suppression (4-5\% lower in comparison with surrounding low latitude areas with similar composition except $\mathrm{H}$ ) at both the north and south poles, interpreted as local water ice rich areas (presumably within permanently shadowed regions) or as a reservoir of volatiles created by solar wind migrate onto cold poles [Feldman et al., 1998b; Crider and Vondrak, 2000; Feldman et al., 2000, 2001; Lawrence et al., 2006]. The best spatial resolution of 
LPNS (full width at half maximum resolution $\sim 46 \mathrm{~km}$ at low altitude about $30 \mathrm{~km}$ [see Maurice et al., 2004]) was too poor to resolve local areas with highest $\mathrm{H}$ abundance leaving open the question of whether the hydrogen was uniformly distributed within the extended suppression regions or localized in permanently shadowed regions (possibly from comet water ice deposition).

[5] Global maps of thermal and fast neutrons also have shown significant regional variations of neutron flux across the surface closely related with non-homogeneity of soil composition. Thus, nearside mare basins and South Pole Aitken (SPA) basin are visible in global maps as strong depressions of thermal neutron flux [see, e.g.,Feldman et al., 1998a; Lawrence et al., 2002]. The highlands on the farside of the Moon, on the contrary, are seen on a global map of thermal neutron flux as high intensity areas, poor in major absorbing elements like Fe and Ti [ Feldman et al., 1998a]. The significant difference in soil composition of nearside mare basins and farside highlands is also clearly visible in the fast neutron global map. It appears as a higher intensity of fast neutron flux in mare basins in comparison with lower intensity in highlands. The major cause of this difference in this case is a variation of average atomic mass of the lunar soil with larger values (more weight fraction of $\mathrm{Fe}$ ) in mare basins and lower values (more weight fraction of Al) in highlands [see Gasnault et al., 2001].

[6] The next mission to implement nuclear methods for studying lunar soil composition appeared in 2009 when another neutron spectrometer, LEND (Lunar Exploration Neutron Detector), was launched into lunar orbit onboard the Lunar Reconnaissance Orbiter (LRO) mission [Chin et al., 2007]. LEND consists of several neutron sensors measuring lunar neutron flux in different energy ranges with different spatial resolutions. It is similar to LPNS in the global monitoring of thermal, epithermal and fast neutrons by omnidirectional neutron detectors, which have spatial resolution strongly limited by the altitude, but LEND has the significant advantage of measuring collimated epithermal neutron fluxes with much better spatial resolution (see Table 1 and Figure 1). LEND physical/geometrical resolution is significantly better than previous measurements made by LPNS. At the low altitude of $\sim 30 \mathrm{~km}$ LPNS claimed resolution of $46 \mathrm{~km}$ measured as full width at half maximum (FWHM). During mapping phase LRO was flying at the altitude of $\sim 50 \mathrm{~km}$. At this altitude LEND can provide FWHM $=10 \mathrm{~km}$. In December $2012 \mathrm{LRO}$ was moved into the frozen elliptical orbit with periapsis about $30 \mathrm{~km}$ above the south pole. It gives possibility to map neutron flux by LEND collimated detectors with spatial resolution $\mathrm{FWHM}=6 \mathrm{~km}$. These estimations were verified through the numerical modeling and calibrations [see, e.g., Mitrofanov et al., 2008, 2010a, 2010b, 2011].

Table 1. The Description of LEND Neutron Detectors

\section{Detector}

\section{Description}

Two ${ }^{3} \mathrm{He}$ proportional counters which are attached to the opposite sides of bottom part of the LEND collimator body. They are oriented along the velocity vector of a spacecraft forming a Doppler pair of detectors. Each detector has efficiency in wide

STN1 and energy range equal approximately to 1 for thermal energies and decaying with energy

STN2 for the epithermal neutrons. The neutron sensor facing forward the spacecraft velocity vector detects significantly more neutrons than detector facing in backward direction. This difference in counting rates strongly depends on neutron's energy having maximum at low thermal energies $(<0.015 \mathrm{eV}-$ equivalent energy for the 
Table 1. The Description of LEND Neutron Detectors

Detector

\section{Description}

neutrons with spacecraft velocity) and fast dropping down to zero for the epithermal energies $(>0.4 \mathrm{eV})$. As result the difference in counting rates is given by thermal neutrons hitting the front side of spacecraft and free of any epithermal neutrons and thermal neutrons background produced in the spacecraft body.

The omnidirectional ${ }^{3} \mathrm{He}$ proportional counter, which is attached to the upper part of STN3 collimator body. This sensor is identical to the Doppler proportional counters having the same sensitivity to the thermal and epithermal neutrons. This detector is used to support the estimation of spacecraft background produced by thermal neutrons.

The omnidirectional ${ }^{3} \mathrm{He}$ proportional counter, which is attached to the upper part of collimator body (opposite side to the STN3). This sensor is covered with $1 \mathrm{~mm}$ sheet of $\mathrm{Cd}$ to stop registration of thermal neutrons with energies below $0.4 \mathrm{eV}$. The data

SETN from this detector are used to provide global mapping of epithermal neutrons with low spatial resolution (full width at half maximum is more than altitude of spacecraft). The difference of counting rates from STN3 and SETN characterizes the thermal component of lunar emission with the energy of neutrons below the $\mathrm{Cd}$ threshold at $0.4 \mathrm{eV}$.

Four ${ }^{3}$ He proportional counters, which are identical to STN1, STN2, STN3 and SETN. Tops of these detectors are open and directed to the nadir and sides are surrounded with polyethelene $-{ }^{10} \mathrm{~B}$ collimator walls, bottom part is covered with cadmium and ${ }^{10} \mathrm{~B}$. It provides the angular efficiency function with half width at half maximum equal to $5 \mathrm{~km}$ at nominal $50 \mathrm{~km}$ altitude. These detectors are primarily used to create polar maps of the Moon with enhanced spatial resolution.

This sensor is a stilben crystal scintillation detector with time separation circuit to split resulted detection signal into gammas and neutrons (registered by recoil protons) channels. This detector is integrated in the center of collimator and enclosed with anticoincidence plastic scintillator to remove «pollution» counting rate produced by

SHEN-N space protons. It detects neutrons with energies from $0.5 \mathrm{MeV}$ up to $15 \mathrm{MeV}$. The collimator walls are not efficient enough to stop fast neutrons as epithermal neutrons, but nevertheless it significantly depresses the flux of high energy neutrons leading to the narrowing field of view of SHEN-N in comparison with omnidirectional detectors. The data from this sensor are used for global mapping of fast neutrons.

Figure 1. Instrument LEND has the set of nine sensors of thermal, epithermal and fast neutrons and collimation module.

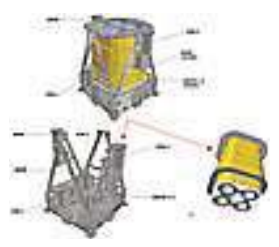

[7] The previous statement should be necessarily combined with the counting statistic measured by LEND. In other words, which portion of the measured counting rate came from the detection 
of epithermal neutrons in the open field of view (FOV) and which is induced by neutrons penetrating through the collimator walls or backscattered in the spacecraft body. It significantly influences the observational (exposure) time needed to distinguish small areas on the Moon surface at the statistically significant level of more than $3 \sigma$. Our analysis (see section 5) has shown that the total counting rate in the collimated detectors is about 5.1 counts per second. It consists of $\sim 1.7$ counts per second measured as a collimated signal and $\sim 3.4$ counts per second resulted from the spacecraft background and penetration through the collimator walls. In our paper, we only focus on the global neutron maps derived from the omnidirectional detectors and do not develop applications of this result to the study of local polar areas. But other publications [Mitrofanov et al., 2010b, 2011] concluded that such proportion in the counting rate is sufficient to distinguish inhomogeneity in the distribution of neutron fluxes at small scale (large polar shadowed areas with $100-1000 \mathrm{~km}^{2}$ ) at least during first year of mapping.

[8] LEND detects neutrons starting from thermal energies (less than $0.015 \mathrm{eV}$ ) up to high energy $(\sim 15 \mathrm{MeV})$ neutrons. The main goals of this paper are to present the first global LEND maps of thermal, epithermal and fast neutrons corrected for various systematic effects; to study regional variations of neutrons in terms of soil elemental composition and to present results of a preliminary comparison with LPNS data.

\section{LEND Instrumentation, Data and Methods}

[9] LEND is a multidetector system consisting of 9 different neutron detectors responsible for registration of neutrons with different energies (for details, see also Mitrofanov et al. [2008, 2010a]). All detectors can be divided into two groups. One is a group of detectors installed inside a heavy polyethylene $-{ }^{10} \mathrm{~B}$ collimator. These detectors have a narrow field of view, which provides the ability to monitor lunar neutron fluxes of epithermal and fast neutrons with improved spatial resolution. Another group of detectors are omnidirectional sensors, which are located outside the collimator body and detect thermal and epithermal neutrons with a spatial resolution defined only by spacecraft altitude $(>75 \mathrm{~km} \mathrm{FWHM}$ at the $50 \mathrm{~km}$ circular orbit during mapping phase). The short description of each LEND detector is presented in the Table 1 and Figure 1.

[10] In section 3 we present data derived from the two omnidirectional proportional counters (STN1 and STN2) mounted on opposite sides of the collimator and aligned along the spacecraft velocity vector [Mitrofanov et al., 2010a]. Combined they act as Doppler pair detectors, sensitive to the registration of thermal neutrons in the energy range below $0.015 \mathrm{eV}$ (see Feldman and Drake [1986] for detailed description of Doppler filter technique proposed for registration of thermal neutrons). Based on these data, we can create a global map of the thermal neutron flux to compare with the LPNS results.

[11] In section 4 we present data derived from the fast neutron detector, which is an organic (stilben crystal) scintillator (SHEN-N detector) with risetime discrimination that splits the signal into gamma and neutron (registered by recoil protons) channels [Mitrofanov et al., 2008, 2010a]. This detector is located in the center of the collimator and surrounded with an anticoincidence plastic scintillator to remove the background counting rate produced by space protons. The whole detector assembly is inherited from the fast neutron detection technique used in the High 
Energy Neutron Detector (HEND), part of the Mars Odyssey instrument suite [ Boynton et al., 2004]. Using this data set we have created a global map of fast neutron flux for comparison with the map provided by LPNS.

[12] In section 5 we concentrate on the comparison of different data sets from omnidirectional SETN detector and four collimated CSETN1-4 detectors with the narrow field of view [Mitrofanov et al., 2010a]. Our goal here is to derive and validate the epithermal component of neutron flux, create global maps and compare with the LPNS map.

[13] All data discussed in section $\underline{3}-\underline{5}$ are taken from the LEND official Planetary Data System (PDS) data for a time period from 15 September 2009 to 15 December 2010, a little longer than the official Exploration Systems Mission Directorate primary mission.

[14] All data sets are passed through the LEND standard data reduction procedures that take into account various systematic effects including correction for efficiency changes, trend of GCRs, exclusion of strong Solar Particle Events, separation between charged particles and neutrons, altitude and temperature correction, and analysis of cruise data to identify and eliminate spacecraft background (see also M. L. Litvak et al., LEND neutron data processing for mapping of the Moon, submitted to Journal of Geophysical Research, 2012).

\section{Distribution of Thermal Neutron Fluxes}

[15] In this section we discuss the main results of global mapping of thermal neutron fluxes measured by LEND Doppler detectors. As it was already mentioned in section 2, for this analysis we have used data from two omnidirectional proportional counters aligned along the spacecraft velocity vector and separated by the LEND collimator. The difference in the counting rates between these two detectors is proportional to the thermal neutron flux at the lowest energies (primarily the neutrons with energies below $0.015 \mathrm{eV}$ ). More than one year of primary mapping data (circular orbit with an average altitude of $50 \mathrm{~km}$ ) was used as input for mapping the thermal neutron flux data. This time period was divided into four shorter time intervals delineated by changing the spacecraft orientation to the velocity vector. The mapping phase began with velocity vector oriented along the spacecraft's $-x$ axis (The $x$ axis can be described as a vector connecting STN2 and STN1 detectors facing in the direction of STN1, see Figure 1). After just two weeks the spacecraft orientation was changed to the opposite one, with velocity vector along the $+x$ axis, where it remained until the end of March 2010, then flipped back to the $-x$ axis. The fourth and final period, with the velocity vector aligned with spacecraft's $+x$ axis, was started at the end of September and lasted up to the end of analyzed period of the mapping. The counting rates in two LEND Doppler detectors, STN1 and STN2, are "velocity direction" dependent with the lead detector measuring thermal and epithermal neutrons and the trailing detector measuring mainly epithermals. The thermal flux for each of the four time periods is the difference of the detectors, STN1-STN2 for the +xorientation and STN2-STN1 for the $-x$ orientation. The data reduction for each Doppler detector included corrections for efficiency and variations in GCR flux. The time profiles of counting rates measured by both omnidirectional detectors SETN and STN3 and all collimated detectors show very similar long-term variations (a slow reduction of counting rates by $\sim 15 \%$ ) during the first year of mapping. It correlates well with the changes of GCR flux observed by GOES and ACE missions. STN1 and STN2 detectors also show the same 
trend of counting rates but are significantly modified by the numerous changes in spacecraft orientation. For detectors STN1, SETN, CSETNs and SHEN-N we have used the time profiles averaged for a long period of time (two weeks) to get corrections factors for the GCR variations (see Litvak et al., submitted manuscript, 2012). For STN1 and STN2 we have used relative trends derived from the averaged STN3 time profile to make correction for the GCR long-term behavior.

[16] Another correction was applied to account for the non-homogeneous distribution of spacecraft mass around the Doppler detectors. The field of view of STN1 is partially obscured by the spacecraft body while the field of view of STN2 is clear of it. This means that some additional background $\mathrm{C}_{\mathrm{bgd} / \mathrm{stn} 1}$ (thermal neutrons from spacecraft) is added to the counting rate of STN1 in comparison with the counting rate of STN2. When the velocity vector is oriented along +xaxis, STN1 is the lead detector and STN2 trails. So the difference in counting rates, STN1-STN2, for this orientation is larger than difference in STN2-STN1, as determined by the spacecraft orientation in the $-x$ axis direction. This is because in the first case, the spacecraft background is added to the difference of counting rates between STN1 and STN2, but in second case it is subtracted making it smaller. Roughly, the background counting rate may be estimated from the comparison of two spacecraft orientations: $2 \times \mathrm{C}_{\mathrm{bgd} / \mathrm{stn} 1}=\left[\left(\mathrm{C}_{\mathrm{stn} 1}-\mathrm{C}_{\mathrm{stn} 2}\right)-\left(\mathrm{C}_{\mathrm{stn} 2}-\mathrm{C}_{\mathrm{stn} 1}\right)\right]$, where $\mathrm{C}_{\mathrm{stn} 1}$ and $\mathrm{C}_{\mathrm{stn} 2}$ are measured counting rates in the detectors STN1 and STN2. Taking this equation into account one may adjust all spacecraft orientation time periods to the standard counting rate in Doppler detectors subtracting $\mathrm{C}_{\mathrm{bgd} / \mathrm{stn} 1}$ from $\mathrm{C}_{\mathrm{stn} 1}$. Following this procedure we estimated counting rate in Doppler pairs for each time interval, and mapped it with resolution $1^{\circ}$ $\times 1^{\circ}$. The resulting statistical uncertainty at each $1^{\circ} \times 1^{\circ}$ pixel depends on the LRO exposure time, which is a function of spacecraft orbiting and instrument on/off periods. The average value of the relative error in each pixel is about $7 \%$. The final map of counting rate is smoothed with a Gaussian filter of FWHM $=60 \mathrm{~km}$, and presented in Figure 2. It is comparable with the spatial resolution of an omnidirectional detector at an altitude of $50 \mathrm{~km}(\mathrm{FWHM} \sim 75 \mathrm{~km})$.

Figure 2. Smoothed (Gaussian filter with FWHM $=60 \mathrm{~km}$ ) map (in Robinson projection) $1^{\circ} \times 1^{\circ}$ of thermal neutron flux from lunar surface measured as a difference in counting rate (counts per second) between pair of LEND Doppler detectors (STN1 and STN2).

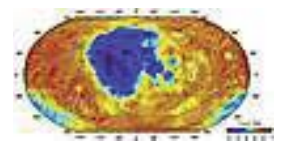

[17] The thermal neutron flux intensity shown on the LEND map has significant dynamic range with a factor 3.5-4 and is reasonably well correlated with the main patterns of lunar soil composition. The major effect is seen on the nearside of the Moon in mare basaltic terrains where there are substantial depressions of thermal neutron flux. In contrast, the highlands on the farside of the Moon show significantly higher intensities of thermal neutron flux. These regional variations have been observed in previous experiments and were analyzed in details using data from LPNS and Lunar Prospector Gamma-Ray Spectrometer (LPGRS) instruments. The correlation of lunar maria with considerable reductions in thermal neutron flux intensity has been interpreted as an enhanced abundance of such primary soil forming elements as Fe and $\mathrm{Ti}$ and minor and trace elements like $\mathrm{Gd}$, and Sm that have large neutron absorption cross sections. Comparison of the distribution of thermal neutron flux detected by LPNS and 7.6 MeV gamma 
ray doublet (Fe neutron capture energy band) counting rates measured by LPGRS has shown that a key factor defining the global distribution of thermal neutrons is iron $(\mathrm{FeO})$ abundance. $\mathrm{FeO}$ abundance has been found to vary from $\sim 5 \%$ by weight in the highlands up to $22-23 \%$ weight in western mare regions with intermediate values $8-10 \%$ at South Pole Aitken basin [see, e.g.,Lawrence et al., 2002]. This pattern correlates very well with the distribution of thermal neutron fluxes measured by the LEND instrument. So, the direct correlation (pixel-to-pixel) between LPNS (taken as special PDS product prepared by LPNS team according to data reduction procedures described inMaurice et al. [2004]) and LEND maps of thermal neutron flux shows very high linear correlation coefficient 0.98 [see, e.g., Press et al., 1992]. It is illustrated in Figure 3.

Figure 3. The correlation between LEND thermal neutron Doppler detectors counting rate ( $y$ axis, counts per second) and LPNS counting rate from thermal neutron detector ( $x$ axis, counts per second) using a $2^{\circ} \times 2^{\circ}$ regular mapping grid. The linear best fit is shown by dashed line.

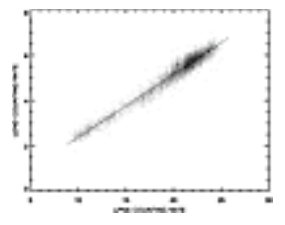

[18] Figure 4 summarizes the results of a comparative analysis of regional variations of thermal neutrons measured on global scale by the LPNS and LEND instruments.

Figure 4. Orbital phase curves (see section 3 ) showing regional latitude variations of thermal neutron flux across the lunar surface. The $x$ axis is the phase angle measured in degrees of arc from $0^{\circ}$ up $360^{\circ}$, where $90^{\circ}$ corresponds to south pole and $270^{\circ}$ to the north pole. The $y$ axis is the normalized counting rate of fast neutrons measured by different instruments. LEND data are shown by solid curve and LPNS data by dashed curve.

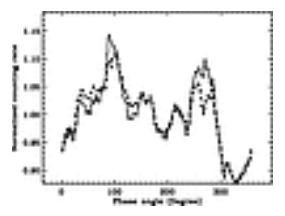

[19] In addition to the global map presented in Figure 2 we can characterize regional variations of neutron flux as a function of latitude. We have combined all orbital measurements into one average orbit, keeping latitude dependence and averaging different longitudes. Such orbital phase profile (OPP) presents a function of phase (latitude) angle $\varphi$ measured in degree of arc from $0^{\circ}$ to $360^{\circ}$ (binning of full orbit around Moon). This is similar to averaging an ensemble of counting rates within wide polar bands, as suggested by Feldman et al. [2001]. In this approach we derive one-dimensional view of the pattern of regional variations presented on the global map (Figure 2) by averaging counting rate across longitudes but leaving its dependence from the latitude. It partially suppresses (large areas like mare basalt terrains still will be quite visible) longitude dependence but significantly increase statistic of counts. Especially it is helpful to search for possible significant variations of neutron flux inside polar regions in comparison with low latitude areas. Our definition of average orbit can be described as follows: The range of 
phase angles $\varphi$ from $0^{\circ}$ to $90^{\circ}$ corresponds to the averaging parts of all available orbits when the spacecraft was moving from equator to south pole within $\left[-180^{\circ}, 0^{\circ}\right]$ longitudes. The range from $90^{\circ}$ to $270^{\circ}$ correspond to averaging all orbits when spacecraft was moving from south pole to north pole within longitudes $\left[0^{\circ},+180^{\circ}\right]$ and finally, $\varphi$ ranges from $270^{\circ}$ to $360^{\circ}$ correspond to the averaging parts of orbits when spacecraft was moving from north pole to equator within longitudes $\left[-180^{\circ}, 0^{\circ}\right]$.

[20] To compare regional variations of thermal neutron fluxes observed by the LPNS and LEND instruments, we have created OPPs and normalized each curve by dividing by the average counting rate. It was done to exclude differences in the absolute values of counting rates measured by different instruments and leave only relative variations in OPP's shape from one lunar region to another. The normalization was performed in accordance with expression $\mathrm{C}_{\text {norm }}=\mathrm{C}_{\text {real }} /\left\langle\mathrm{C}_{\text {real }}\right\rangle$, where $\mathrm{C}_{\text {real }}$ is the absolute value of counting rate measured by one of the instruments and $\left\langle\mathrm{C}_{\text {real }}\right\rangle$ is its mean value calculated for the whole lunar surface. From Figure 4 it is seen that the normalized LEND and LPNS OPPs have similar variations. Discrepancies are most visible in the polar and southern intermediate latitude regions. Poleward of $70^{\circ}$ both in the south and north, the LEND profile shows higher values (in comparison with values observed at equatorial regions) of thermal neutron flux than in the LPNS data. At the intermediate southern latitude belt (30S-75S) the thermal flux measured by LEND is lower than that from LPNS .

[21] We suggest that a possible explanation of the polar differences lies in the latitude variation of the lunar subsurface temperature. LPNS measured thermal neutron fluxes in the energy range below the cadmium threshold $(<0.4 \mathrm{eV}$ [see Feldman et al., 2004]) derived from the difference between counting rates from a bare ${ }^{3} \mathrm{He}$ proportional counter and one covered with $0.63 \mathrm{~mm}$ of $\mathrm{Cd}$. The primary method for detection of thermal neutron fluxes by LEND is based on the Doppler technique, measuring thermal neutrons with considerably less energies (mostly below $0.015 \mathrm{eV}$ ) than those measured by LPNS. Little et al. [2003] and Lawrence et al. [2006] have concluded that thermal neutron fluxes measured by LPNS are temperature dependent and vary with soil type. It was verified with numerical simulations that thermal neutron fluxes in this energy range increase with increasing temperature of the soil, leading to the maximum variations between equator $(\sim 250 \mathrm{~K})$ and polar latitudes $(\sim 100 \mathrm{~K})$ of about $5 \%$. Thermal neutrons detected by LEND belong to the low energy tail of the Maxwellian distribution and follow an opposite temperature trend. With increasing temperature the peak of the Maxwellian distribution is shifted to higher energies. It decreases the number of neutrons in the energy range below $0.015 \mathrm{eV}$ (where Doppler detector efficiency is maximum). This leads to decreasing counting rates with increasing soil temperature. As a result, if the normalized LPNS and LEND counting rates fit each other at the equatorial regions (with high surface temperature) then at polar latitudes (with lower temperatures) we should expect discrepancies in their regional variations: larger amplitude of variations for LEND (increased by temperature effect) and smaller one for LPNS (decreased by temperature effect). Preliminary simulations of LEND data show that temperature variations may be as large as 5-8\%. In this paper we have not included a quantitative correction of the thermal neutron counting rates (measured by Doppler detectors) for soil temperature. It is a second order effect, much smaller than global regional variations of the thermal neutron flux. One can compare possible temperature induced variations $<10 \%$ with full scale of regional variations of $300-400 \%$ presented on the map in Figure 2. As a result this effect does not 
significantly alter the main patterns of the global map of lunar thermal neutron flux (high pixelto-pixel linear correlation coefficient between LPNS and LEND maps shown in Figure 3).

[22] For southern moderate latitudes (30S-75S) we hypothesize that differences between LEND and LPNS OPPs are due to variations of soil composition and different sensitivity of neutrons with energies $<0.015 \mathrm{eV}$ (LEND Doppler filter) and neutrons with energies $<0.4 \mathrm{eV}$ (LPNS omnidirectional detectors) to detect such soil variations.

[23] To investigate differences between LEND and LPNS OPPs at equatorial and intermediate latitudes in more detail we have performed pixel-to-pixel comparison of normalized (to adjust different instrument sensitivities) LEND and LPNS counting rates. The result of the comparison is presented as a global map of a value $\mathrm{D}$ of pixel-to-pixel difference. The value $\mathrm{D}_{\mathrm{ij}}$ at each pixel $\{i, j\}$ is defined as:

$$
\text { - } D_{i j}=\frac{\left|\frac{L E N D_{i}}{\langle L E N D\rangle}-\frac{L P N S_{i j}}{\langle L P N S\rangle}\right|}{\delta_{i j}}
$$

where $\operatorname{LEND}_{\mathrm{ij}}$ is the LEND counting rate at the pixel $\{\mathrm{i}, \mathrm{j}\},<\mathrm{LEND}>$ is the LEND average counting rate (calculated for the whole lunar surface), LPNS $\mathrm{ij}_{\mathrm{ij}}$ is the LPNS counting rate at the pixel $\{\mathrm{i}, \mathrm{j}\},<\mathrm{LPNS}>$ is the LPNS average counting rate (calculated for the whole lunar surface) and finally $\delta_{\mathrm{ij}}$ is the total statistical error of the numerator in equation (1) deduced from the statistical uncertainties of LEND and LPNS counting rates at the pixel $\{\mathrm{i}, \mathrm{j}\}$.

[24] Conceptually the definition of $\mathrm{D}_{\mathrm{ij}}$ is close to the definition of signal-to-noise ratio (SNR) showing how the value of signal is statistically different from the background. In our case the difference between normalized LEND and LPNS counting rates (nominator of (1)) is used as a "signal" and the statistical error of this difference (denominator of (1)) is a measure of "signal" significance. The value of $\mathrm{D}_{\mathrm{ij}}$ presents a number of statistical deviations (sigmas) between LEND and LPNS data. If it exceeds 3 sigma level the difference between LEND and LPNS in the given pixel can be considered as significant. The resulted map of $\mathrm{D}_{\mathrm{ij}}$ is shown in Figure 5. The yellow and red colors on this map correspond to the most significant differences between LEND and LPNS regional variations. It is seen that such "discrepancy" areas are distributed at the poles (already discussed above) and at the equatorial and moderate latitudes with enhanced content of $\mathrm{Fe}$ (mostly at South Pole Aitken basin).

Figure 5. The map of comparison (number of statistical deviations) between LEND thermal neutron counting rate (pair of Doppler detector) and thermal neutron counts from LPNS detector. For details, see section 3 , equation (1).

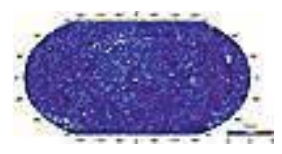

[25] We may conclude that the difference between LPNS and LEND regional variations of thermal neutron fluxes at mid-southern latitudes is produced by a different sensitivity of thermal neutrons with different energies due to the variations of soil composition. 


\section{Distribution of Fast Neutron Fluxes}

[26] In this section we present results of global mapping of the fast neutron fluxes measured by LEND SHEN-N detector. Here we have used data from the organic scintillator stilben $\left(\mathrm{C}_{6} \mathrm{H}_{5} \mathrm{CH}=\mathrm{CHC}_{6} \mathrm{H}_{5}\right)$ located inside the collimator (for details, see Mitrofanov et al. $[2008$, 2010a]). This detector is used for detection of high energy neutrons by recoil protons in the stilben crystal lattice. The incident neutron loses its energy, E, through the collision of hydrogen nuclei leading to a nearly random and uniform spectrum of protons in the energy range from 0 to E. The LEND collimator is not as effective in stopping fast neutrons as it is for the epithermal neutrons, but it nevertheless narrows the SHEN-N field of view. The real spatial resolution of the detector measured as full width at half maximum is about $40 \mathrm{~km}$. SHEN-N data are measured as a 16 channel spectrum of counting rate induced by fast neutrons in the energy range from $\sim 500 \mathrm{keV}$ up to $15 \mathrm{MeV}$. To improve statistics we combine all higher channels (\#10-15) excluding low energy neutrons near the detector threshold (to exclude thresholds systematic effects and low statistic channels) and the highest energy integration channel (\#16), which sums all counts from recoil protons with energies above $10 \mathrm{MeV}$ (to exclude very energetic neutrons which are insensitive to the soil composition).

[27] It is known [e.g., Feldman et al., 1998a] that fast neutron flux is an important component of the lunar neutron environment, is sensitive to the soil composition, and may be used to infer average elemental composition. Multiple publications have confirmed the linear relation between fast neutron flux and average atomic mass of soil composition, using both experimental data and numerical simulations [Maurice et al., 2000; Gasnault et al., 2001].

[28] We have produced the LEND global map of distribution of counting rate from the fast neutron detector with a resolution of $1^{\circ} \times 1^{\circ}$ degree. Pixels on the map have varied statistical uncertainty depending on the LRO exposure time. The mean relative error is about $11 \%$. As in case of thermal neutrons map we have smoothed fast neutrons map by Gaussian filter with FWHM equal to $60 \mathrm{~km}$ (SHEN-N has better spatial resolution but significantly less counting statistics). The result is presented in Figure 6. It clearly shows a non-homogeneous distribution of fast neutron flux across the lunar surface. The basaltic mare terrains at the low latitudes are seen as bright areas with substantially higher neutron flux than those observed in the highlands. The same pattern of fast neutrons is also seen in the LPNS data [ Feldman et al., 1998a]. The observed effect is explained as a correlation of fast neutron flux with average atomic mass: in mare regions enriched with $\mathrm{Fe}$ and $\mathrm{Ti}$ the average atomic mass of the soils is larger than in the highlands ( $\mathrm{Al}$ and $\mathrm{Ca}$-dominating elements). To confirm this conclusion we have compared the average atomic mass calculated as equation (2) for known elemental compositions of lunar landing sites for Apollo/Luna missions and LEND counting rate of fast neutron detector.

$$
\langle A\rangle=\frac{1}{\sum_{n} w_{n} / A_{n}}
$$

where $A_{n}$ its atomic mass of element $n$ (in atomic mass units, amu), and $w_{n}$ its weight fraction. In Figure 7 one can see the results of such analysis. The $x$ axis on this figure corresponds to the LEND counting rate derived for each landing site (smoothed counting rate in pixel $1^{\circ} \times 1^{\circ}$ centered on a landing site). The $y$ axis corresponds to the average atomic mass calculated by 
equation (2) for each landing site. A combination of Apollo and Luna mission landing sites compositions was used [see, e.g., Vinogradov et al., 1966; Metzger et al., 1973; Lawrence et al., 2006]. All eight data points (Apollo 11, Apollo 12, Apollo 15, Apollo 16, Apollo 17, Luna 16, Luna 20, Luna 24; the same set of data as analyzed in Gasnault et al. [2001]) are well described by a simple linear fit (see the solid curve in Figure 7). The linear correlation coefficient [ $\underline{\text { Press et }}$ al., 1992] is considerably high, estimated to be $\sim 0.9$. The possible deviations from the linear law are explained by large statistical errors of the counting rate, poor spatial resolution of the SHEN$\mathrm{N}$ (much larger than the landing site area) and uncertainties in the estimation of elemental composition.

Figure 6. Smoothed (Gaussian filter with FWHM $=120 \mathrm{~km}$ ) map (in Robinson projection) $1^{\circ} \times$ $1^{\circ}$ of fast neutron flux (in counts per second) from the lunar surface measured by LEND SHEN$\mathrm{N}$ detector.

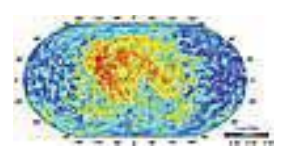

Figure 7. Average atomic mass of the sampled lunar soils versus fast neutron counting rate measured by LEND for the landing site regions. The best fit is shown by dashed line.

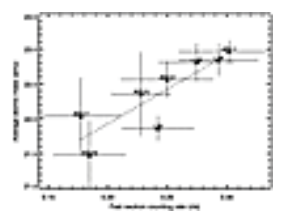

[29] To compare LEND data and LPNS data, as was done for the thermal neutrons, we also have used latitude orbital phase profiles and estimated a correlation coefficient. The pixel-to-pixel correlation between LEND and LPNS maps is shown in Figure 8. The linear correlation coefficient derived from this analysis is equal to $\sim 0.6$. It is significantly high value taking into account low counting statistics of SHEN-N and the small regional variations of neutron flux everywhere except Fe enriched areas. The comparison of orbital phase profiles (OPPs) was also done and presented in Figure 9. To create LEND and LPNS OPPs (we used LPNS map of fast neutrons provided in the special PDS data product based on the data reduction procedure described in Maurice et al. [2004]) we used the technique described in section 3. The LEND and LPNS orbital phase profiles follow each other. The small differences between them are covered by $1-\sigma$ uncertainty of the LEND/LPNS normalized counting rates. Both OPPs show significant increases in counting rates $(10 \%$ above average counting rate) at equatorial and moderate latitudes, corresponding to predominantly mare terrains, and lower counting rates in the highlands, confirming the correlation of fast neutron flux with average atomic mass distribution. At polar latitudes we do not see any substantial neutron suppression, as it is observed in epithermal neutrons (see next section). The comparison of epithermal and fast neutron fluxes distributions may be used for the verification of depth dependent distribution of Hydrogen at lunar poles. It may be done through detailed numerical modeling and use of a two layer model with an upper dry layer (with variable thickness) and a bottom layer with variable hydrogen concentration (probably in the form of dirty water ice). In analogy with Mars neutron data 
analysis, the HEND instrument integrated onboard the Mars Odyssey spacecraft [see, e.g., Litvak et al., 2006] shows that the thickness of the upper dry layer may be as large as $20 \mathrm{~cm}$.

Figure 8. The correlation between LEND SHEN-N fast neutron counting rate $(y$ axis, counts per second) and LPNS counts from fast neutron detector ( $x$ axis, counts per second) using a $2^{\circ} \times 2^{\circ}$ regular mapping grid. The linear best fit is shown by dashed line.

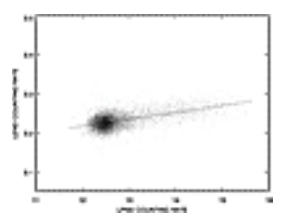

Figure 9. Orbital phase curves (see section 4) showing regional latitude variations of fast neutron flux across the lunar surface. The $x$ axis is the phase angle measured in degrees of arc from $0^{\circ}$ up $360^{\circ}$, where $90^{\circ}$ corresponds to the south pole and $270^{\circ}$ to the north pole. The $y$ axis is the normalized counting rate of fast neutrons measured by different instruments. LEND data are shown with a solid curve and LPNS data with a dashed curve.

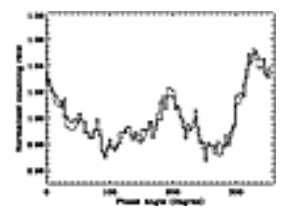

\section{Distribution of Epithermal Neutron Flux}

[30] In Figure 10 we show the map of counting rate detected by collimated sensors CSETNs (see Table 1). Counting rates presented on this map are corrected for the detector efficiency changes (smooth saturation of counting rate after multiple switch offs/ons required by a correction of LRO orbit each 2 weeks) and for a long-term variations of GCR (variations of GCR flux as a function of solar cycle induce corresponding variations of Moon neutron flux). We also have taken into account a spacecraft background induced by the bombardment of the spacecraft body with particles of GCRs. The total value of background was estimated at the cruise stage far away from the Moon using the LEND measurements in the same configuration (detectors levels of high voltage, thresholds) as was set for the mapping phase. Then this value was adjusted for the LRO orbit (Moon partially shields spacecraft from the irradiation by GCRs leading to decreasing of the background) and subtracted it from the measurements. So, the result of the data processing presented in Figure 10 shows distribution of Moon neutrons only and logically can be divided into three different components. First one is the neutrons came into collimator FOV, second is the omnidirectional neutrons passed through collimator walls and finally third component presents omnidirectional neutrons backscattered in the spacecraft body.

Figure 10. Smoothed (Gaussian filter with FWHM $=120 \mathrm{~km}$ ) map (in Robinson projection) $1^{\circ} \times$ $1^{\circ}$ of counting rate (background subtracted) measured in LEND collimated detectors. 


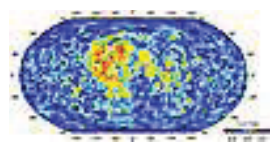

[31] The map shows several distinctive regions with considerable deviations from the average counting rate (value averaged along all map pixels). First, polar regions display a depression of counting rate due to enhancement of hydrogen abundance at high latitudes (first revealed by LPNS). Second, at the nearside equatorial mare regions LEND CSETNs count significantly more neutrons than at the mid latitudes on the farside of the Moon. The corresponding quantitative latitude changes of CSETNs counting rate are presented in Figure 11 as a normalized orbital phase profile (same procedure as was used for thermal and fast neutrons in previous sections). At the poles it shows a $3-4 \%$ depression below average level of counting rate. On the contrary, at mare basins on the nearside of the Moon one can see 2-3\% excess above average level of counting rate (phase angles $300^{\circ}-360^{\circ}$ in Figure 11). We may conclude that spatial distribution of counting rate measured by LEND collimated detectors differs from the global maps created using data of omnidirectional neutron detectors. LPNS [see, e.g., Feldman et al., 2001; Maurice et al., 2004] and LEND (SETN detector, see for description Table 1 and its normalized OPP in Figure 11) omnidirectional epithermal neutron detectors show less neutron counts at both poles ( $\sim-5 \%$ of depression) and no significant excess of counting rate at the nearside mares (phase angles $300^{\circ}-360^{\circ}$ in Figure 11).

Figure 11. Uncorrected orbital phase curves (see section 5) showing regional latitude variations of epithermal neutron flux across the lunar surface measured by LEND omnidirectional SETN (dash-dot line) and collimated CSETNs (solid line) detectors. The $x$ axis is the phase angle measured in degrees of arc from $0^{\circ}$ up $360^{\circ}$, where $90^{\circ}$ corresponds to the south pole and $270^{\circ}$ to the north pole. The $y$ axis is the normalized counting rate of epithermal neutrons.

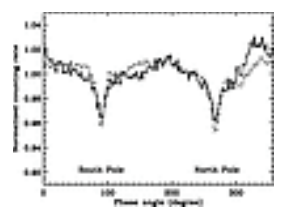

[32] In summary, we may conclude that the distribution of counting rates measured by LEND collimated detectors does not fully correlate with the distribution of epithermal neutron flux measured by omnidirectional neutron detectors. These distributions are similar at the poles showing evident suppression of counting rates but at midlatitudes on the nearside of the Moon, LEND collimated detectors count more neutrons than it is predicted from the omnidirectional detectors of epithermal neutrons (see Figure 11).

[33] Lawrence et al. [2011] have proposed that high energy epithermal and fast neutrons may pass through the collimator losing energy and shifting to the energy range where it may be detected by ${ }^{3} \mathrm{He}$ tubes. Using relative variations of fast neutrons measured by LPNS they suggested that detection of high energy neutrons may add up to 2.25 counts per second into the total counting rate of the LEND collimated detectors. Using the same technique, Mitrofanov et al. [2011] have argued against this estimation and have shown that major "pollution" is contributed by fast neutrons and may be found as 1.1 counts per second. Mitrofanov et al. [2011] also have implemented more comprehensive approach comparing relative latitude variations 
(orbital phase profiles) of neutrons with different energies. It was done to understand how thermal, epithermal and fast neutrons latitudes variations should be mixed to determine latitude variations for counting rate observed in the collimated detectors. Following this approach it was again confirmed that penetration of fast neutrons describes the distribution of counting rates in the collimated detectors and amplitude of this component in the total counting rate is about 1.1 counts per second.

[34] In this paper we continue this analysis to derive a true global map of lunar epithermal neutron flux.

[35] The basic idea of this method postulates that collimated and omnidirectional detectors (based on identical ${ }^{3} \mathrm{He}$ tubes) may count different absolute numbers of neutrons (due to different FOVs) but relative variations of their counting rates should follow the same latitude profile shape. For example, if signal in a collimated detector changes by $\sim 5 \%$ between latitude 1 and latitude2 (and the difference between these latitude belts is much larger then collimated FOV) we can predict the same relative change in the signal of the omnidirectional detector. It is obvious that the last statement is true only if both detectors count neutrons in the same energy range. The latitude variations for collimated and omnidirectional detectors can be presented as orbital phase profiles normalized to the average counting rate measured by each detector. Applying OPP for the epithermal LEND data sets (CSETNs and SETN) we could expect that $\operatorname{OPP}_{\mathrm{CSETNs}}(\varphi)$ was equal to the $\operatorname{OPP}_{\operatorname{SETN}}(\varphi)$. In reality, as we already established (see Figure 11), their shapes are quite different.

[36] To explain this difference we propose that the total counting rates in CSETNs and SETN may also include contributions from thermal, high-energy epithermal and fast neutrons. The CSETNs are well protected from thermal neutrons (by collimator body, additional cadmium and ${ }^{10} \mathrm{~B}$ sheets on the detectors tops) but it may be sensitive for the high-energy epithermal and fast neutrons moderated in the collimator walls or backscattered in the spacecraft body.

Comparing latitude variations of CSETNs and SETN (presented in Figure 11) one may state that "non-epithermal" component in the CSETNs total counting rate both increase neutron flux at the poles (because poles depressions of CSETNs curve are significantly smaller than SETN ones) and at the equatorial latitudes at the nearside mares (CSETNs curve lies above SETN one at the range of phase angles $300^{\circ}-360^{\circ}$ ). The best model of such "non-epithermal" component is the latitude variations of the fast neutrons measured by LEND SHEN-N detector (see section 4 ). According to Figure 9 the OPP for SHEN-N detector has the significant increase of counting rate at the midlatitudes (phase angles $300^{\circ}-360^{\circ}$ ) and negligible depression at the poles.

[37] On the other side, SETN (like LPNS epithermal detector) is not sensitive to the high energy and fast neutrons [see, e.g., Lawrence et al., 2011] but may detect some small portion of the thermal neutrons leaking through the cadmium enclosure of SETN.

[38] Concluding this discussion we may say the following: To attain the true counting rate of epithermal neutrons in the collimated detectors we need to subtract the unknown fraction of counting rate from the detection of fast neutrons that propagate through the collimator walls or backscattered in the spacecraft body. The latitude behavior of this "background" component is described by OPP SHEN-N. To get the true counting rate of epithermal neutrons in the 
omnidirectional detector SETN we possibly need to subtract the unknown fraction of the counting rate deposited by thermal neutrons. The latitude behavior of this component can be described by OPP ${ }_{\text {Doppler }}$ described in the section 3 (see Figure 4).

[39] Mathematically it may be presented as two equations:

$$
\begin{aligned}
& \text { - } O P P_{C S E T N s}^{\text {Total }}=(1-\alpha) \times O P P_{C S E T N s}^{\text {Epithermal }}+\alpha \times O P P_{S H E N-N}^{\text {Fast }} \\
& \text { - } O P P_{S E T N}^{\text {Total }}=(1-\beta) \times O P P_{S E T N}^{\text {Epithermal }}+\beta \times O P P_{\text {Dopplers }}^{\text {Thermal }}
\end{aligned}
$$

where OPP ${ }^{\text {total }}{ }_{\text {CSETNs }}$ and OPP ${ }^{\text {Total }}$ SETN are relative latitude variations measured by CSETNs and SETN (presented in Figure 11); $\alpha$ and $\beta$ are free parameters; OPP SHEN-N $^{-}$normalized counting rate derived from LEND fast neutron detector SHEN-N (see section 4 and Figure 9) and $\mathrm{OPP}_{\text {Dopplers }}$ is a difference of counting rate measured by the Doppler detectors presenting global variations of thermal neutrons (see section 3 and Figure 4). OPP ${ }^{\text {Epithermal }}{ }_{\text {CSETNs }}$ and

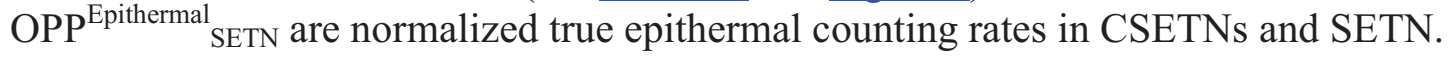
Postulating that they must be equal each to the other, we may solve the system of equations and find the best fit values of $\alpha$ and $\beta$ for the whole range of phase angles, $\varphi$. Quality of this fit is verified by a Pearson criteria test based on a least squares analysis. We have used expression

$$
S^{2}=\sum_{\phi_{i}} \frac{(A(\alpha)-B(\beta))^{2}}{\delta^{2}}
$$

where A is a OPP ${ }^{\text {Epithermal }}{ }_{\text {CSETNs }}$ expressed from equation (3) as a function of $\alpha, \mathrm{B}$ is $\mathrm{OPP}^{\text {Epithermal }}{ }_{\text {SETN }}$ expressed from equation (4) as a function of $\beta, \delta$ - is a total uncertainty derived from the statistic of counts in CSETNs, SETN, Dopplers and SHEN-N detectors, $\left\{\phi_{\mathrm{i}}\right\}-$ set of phase angles. Varying $\alpha$ and $\beta$ we may minimize $\mathrm{S}^{2}$. According Pearson criteria if difference A-B agrees within statistical uncertainties then $\mathrm{S}^{2}$ shall follow distribution $\chi^{2}$ with $\mathrm{N}$ degree of freedom where $\mathrm{N}$ is equal to number of summands in sum (5) reduced by number of free parameters [Press et al., 1992].

[40] We have discovered that it is possible to find such values of parameters $\alpha$ and $\beta$, which will be accepted by Pearson criteria. It confirms that suggested division on the components was reasonable. The results of this analysis are given in Figure 12 where we have shown corrected OPP for the collimated detectors (corrected for high energy/fast neutrons) and OPP for the omnidirectional detector SETN(corrected for the thermal neutrons). This analysis leads us to an estimation of the best fit values of fractions of "true epithermal neutrons" and "non-epithermal neutrons" measured by the collimated and omnidirectional detectors. So, the resulting counting rate in the collimated detectors produced only by epithermal neutrons in the FOV is equal to $\sim 1.7$ counts per second, in good agreement with our previous estimates [Mitrofanov et al., 2010a, 2010b, 2011; R. S. Sagdeev et al., Use of Apollo 17 epoch neutron spectrum as a benchmark for the Collimated 1 Sensors of the Lunar Exploration Neutron Detector, submitted to Journal of Geophysical Research, 2012]. The counting rate of epithermal neutrons in SETN is about 9.8 counts per seconds. Farther we will use this signal to present a global map of epithermal neutrons. 
Figure 12. Corrected orbital phase curves (see section 5) showing regional latitude variations of epithermal neutron flux across the lunar surface measured by LEND omnidirectional SETN (dash-dot line) and collimated CSETNs (solid line) detectors. The $x$ axis is the phase angle measured in degrees of arc from $0^{\circ}$ up $360^{\circ}$, where $90^{\circ}$ corresponds to the south pole and $270^{\circ}$ to the north pole. The $y$ axis is the normalized counting rate of epithermal neutrons.

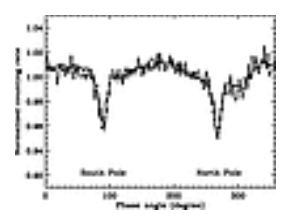

[41] Figure 13 shows OPP for the SETN signal corrected for the "non-epithermal" component (same as in Figure 12) and OPP created from the LPNS PDS maps data sets (special data product), but with the following difference: the epithermal neutron counting rate is reduced by $\varepsilon^{*}$ thermal neutrons, where $\varepsilon$ is equal to 0.057 as recommended in Feldman et al. [2001]. Comparing these OPPs one may conclude that the shape and amplitude of neutron extended suppression measured by LEND around the lunar poles agrees with the LPNS data. But one can see that at equatorial highlands on the farside of the Moon, the LEND average counting rate is higher than that given by LPNS. At moderate latitudes in the north, LPNS predicts a higher intensity of epithermal neutron flux compared to LEND data. It can be seen in more detail in Figure 14 where we have shown a map of relative differences between LEND and LPNS epithermal neutron fluxes expressed in the statistical deviations (same approach as used in the $\underline{\text { section } 3}$, equation (1)). The yellow and red areas on this map correspond to maximal discrepancies between LEND and LPNS data. The largest discrepancies between LEND and LPNS are seen in the northern hemisphere at Mare Frigoris where LPNS has significantly higher values of epithermal flux than LEND and at equatorial latitudes $[-30 \mathrm{~S}, 30 \mathrm{~N}]$ in the highlands on farside (with longitudes from $-180^{\circ}$ up to $-120^{\circ}$ and from $120^{\circ}$ up to $180^{\circ}$ ) of the Moon where in contrast LEND is counting more epithermal neutrons than LPNS. In Figure 15 we also have presented LEND/LPNS pixel-to-pixel correlation. It shows fairly well linear correlation coefficient is about 0.73 . It is easily seen (in Figure 14) that the best agreement between LEND and LPNS data is in the southern hemisphere poleward of $\sim 50 \mathrm{~S}$ for all longitudes. In the north there is a good agreement near polar latitudes (the same effect of extended polar suppression). The significant correlation is also found poleward of $60 \mathrm{~N}$ within $\left[-180^{\circ},-120^{\circ}\right]$ and $\left[60^{\circ}, 180^{\circ}\right]$ longitude sectors. The same pattern of distribution of low values of epithermal flux is seen at moderate and near equatorial latitudes in the vicinity of Mare Imbrium basin.

Figure 13. Corrected orbital phase curves (see section 5) showing regional latitude variations of epithermal neutron flux across the lunar surface measured by LEND omnidirectional SETN (dash-dot line) and LPNS (solid line) detectors. The $x$ axis is the phase angle measured in degrees of arc from $0^{\circ}$ up $360^{\circ}$, where $90^{\circ}$ corresponds to the south pole and $270^{\circ}$ to the north pole. The $y$ axis is the normalized counting rate of epithermal neutrons.

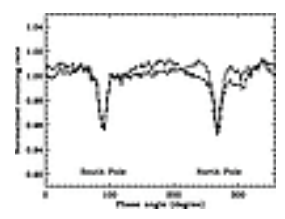


Figure 14. The map of comparison (number of statistical deviations) between LEND epithermal neutron counting rate (SETN detector) and epithermal neutron counts from LPNS detector. For details, see section 5 and equation (1).

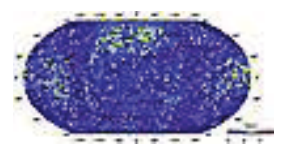

Figure 15. The correlation between LEND corrected epithermal neutron counting rate (SETN, $y$ axis, counts per second) and LPNS counts from epithermal neutron detector ( $x$ axis, counts per second) using a $2^{\circ} \times 2^{\circ}$ regular mapping grid. The counting rate in SETN was corrected for the detector efficiency changes, long-term variations of GCRs. The counting rate was also reduced by the GCR spacecraft background (defined at the cruise) and by contribution of thermal neutron counting rate derived from equations (3) and (4). The linear best fit is shown by dashed line.

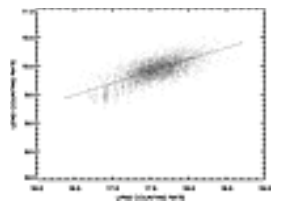

[42] The resulting corrected global and polar maps of LEND epithermal counting rate (as measured by omnidirectional sensor with spatial resolution, $\sim 75 \mathrm{~km}$ and reasonably good counting statistics, average relative error $<2 \%$ ) are shown in Figures 16 and 17 . They are done with a $1^{\circ} \times 1^{\circ}$ grid and smoothed with a Gaussian filter with FWHM equal to $60 \mathrm{~km}$ (compared with omnidirectional spatial resolution). Several large crater basins both in the farside and nearside show increasing intensity of epithermal neutron flux in comparison with neighboring areas. For example, one can see a substantial increase of epithermal neutron flux in the southern hemisphere in such crater basins as Clavius, Maginus, Longomontanus and Tycho (marked in Figure 16). At the equatorial latitudes within $[-30 \mathrm{~S}, 30 \mathrm{~N}]$ high epithermal flux is observed at Mare Orientale, Mare Fecunditatis, Mare Nectaris, Mare Crisium and Mare Moscovience.

Figure 16. Smoothed (Gaussian filter with FWHM $=60 \mathrm{~km}$ ) map (in Robinson projection) $1^{\circ} \times$ $1^{\circ}$ of epithermal neutron counting rate (background subtracted, thermal neutron component removed, counts per second) measured in LEND SETN detector. The counting rate in SETN was corrected for the detector efficiency changes, long-term variations of GCRs. The counting rate was also reduced by the GCR spacecraft background (defined at the cruise) and by contribution of thermal neutron counting rate derived from equations (3) and (4).

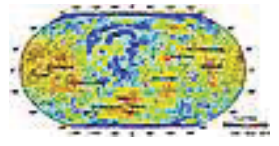

Figure 17. (top) Smoothed (Gaussian filter with FWHM $=60 \mathrm{~km}$ ) map (in stereographic projection) $1^{\circ} \times 1^{\circ}$ poleward of $70 \mathrm{~S}$ of epithermal neutron counting rate (background subtracted, thermal neutron component removed, counts per second) measured in LEND SETN detector. The counting rate in SETN was corrected for the detector efficiency changes, long-term variations of GCRs. The counting rate was also reduced by the GCR spacecraft background 
(defined at the cruise) and by contribution of thermal neutron counting rate derived from equations (3) and (4). (bottom) Smoothed (Gaussian filter with FWHM $=60 \mathrm{~km}$ ) map (in stereographic projection) $1^{\circ} \times 1^{\circ}$ poleward of $70 \mathrm{~N}$ of epithermal neutron counting rate (background subtracted, thermal neutron component removed, counts per second) measured in LEND SETN detector. The counting rate in SETN was corrected for the detector efficiency changes, long-term variations of GCRs. The counting rate was also reduced by the GCR spacecraft background (defined at the cruise) and by contribution of thermal neutron counting rate derived from equations (3) and (4).

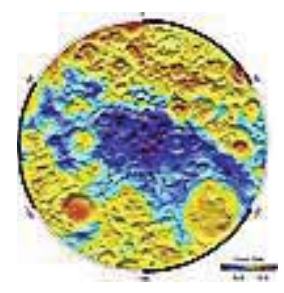

[43] Polar maps from omnidirectional detectors cannot help us to distinguish correlations of neutron suppression with distribution of polar permanently shadow craters due to poor spatial resolution. But polar maps of epithermal neutron flux measured in LEND collimated sensors can be created on a much finer grid. These maps may be used to search for local areas with counting rates showing significant neutron suppression or excess. The detailed discussion concerning such an analysis is given in I. G. Mitrofanov et al. (Testing polar spots of water-rich permafrost on the Moon: LEND observations onboard LRO, submitted toJournal of Geophysical Research, 2012); W. V. Boynton et al. (High spatial resolution of epithermal neutron emission from the lunar poles: Constraints on hydrogen mobility, submitted to Journal of Geophysical Research, 2012; and Sanin et al. [2012] (distribution of epithermal neutron flux inside and outside Polar Shadowed Regions).

\section{Summary}

[44] We have presented the latest results of global mapping of lunar neutron flux measured by the LEND instrument onboard the LRO mission. Three neutron energy ranges, thermal $(<0.015 \mathrm{eV})$, epithermal $(0.4-500 \mathrm{eV})$ and fast $(>0.5 \mathrm{MeV})$ have been studied in our analysis. Thermal neutrons are sensitive to specific elements in the lunar subsurface with high absorption cross sections (like $\mathrm{Fe}$ ). Hydrogen is a dominant factor in determining the variations of epithermal neutron flux. The changes of fast neutron flux mainly follow the average atomic mass of lunar regolith. Global maps of derived (corrected for systematic effects) counting rates have been separately created for thermal, epithermal and fast neutron energy ranges. The regional variations observed on these maps are reasonably well correlated on a global scale with previous (LPNS) results, but show differences on a local scale.

[45] The largest regional variations (up to a factor of 3-4) are seen in the LEND map of thermal neutrons counts between the Fe-rich mare basaltic terrains on the nearside and Fe-poor highlands on the farside of the Moon (see Figure 2). It is similar to the LPNS results [see, e.g., Lawrence et al., 2002]. 
[46] The variation of the epithermal neutron flux across the lunar surface (SETN map, Figure 16) is much smaller $(\sim 10 \%)$ and visible in both LEND and LPNS data as the extended neutron suppression poleward of $70 \mathrm{~S}$ and $70 \mathrm{~N}$. At moderate and equatorial latitudes (where observed regional variations are much smaller) the differences between LEND and LPNS maps are most noticeable. It may be a result of counting statistics or different approaches in data reduction procedures.

[47] The fast neutron flux changes by $25 \%$ across the lunar surface from maria to the highlands, with the maximum of fast neutron flux observed in mare terrains. This result is consistent with the LPNS argument that mare basalts are rich in Fe (producing more fast neutrons in comparison with Al-rich soils). The latitude band profile of fast neutron flux does not show a significant polar extended neutron suppression effect as observed in the epithermal neutron range. One may distinguish only small areas around poles with neutron suppression less than 1\%. By comparison to detection of water ice at Mars polar latitudes by HEND [see, e.g.,Litvak et al., 2006], this may be interpreted as the hydrogen distribution at the lunar poles, on average, is depth dependent with higher weight fraction of $\mathrm{H}$ beneath a relatively hydrogen poor regolith.

\section{Acknowledgments}

[48] We wish to thank the International Space Science Institute (ISSI, Bern, Switzerland) for the support of research (included in the framework of international team "Nuclear Planetology" in 2007-2010) presented in this paper.

\section{References}

- Boynton, W. V., et al. (2004), The Mars Odyssey Gamma-Ray Spectrometer instrument suite, Space Sci. Rev., 110(1-2), 37-83, doi:10.1023/B:SPAC.0000021007.76126.15.

- CrossRef,

- $\underline{\text { CAS }}$,

- Web of Science® Times Cited: 98,

- $\mathrm{ADS}$

- Go here for SFX

- Chin, G., et al. (2007), Lunar reconnaissance Orbiter overview: The instrument suite and mission, Space Sci. Rev., 129, 391-419, doi:10.1007/s11214-007-9153-y.

- CrossRef,

- Web of Science ${ }^{\circledR}$ Times Cited: 69,

- $\underline{\text { ADS }}$

- Go here for SFX

- Crider, D. H., and R. R. Vondrak (2000), The solar wind as a possible source of lunar polar hydrogen deposits, J. Geophys. Res., 105(E11), 26,773-26,782, doi:10.1029/2000JE001277.

Direct Link:

- Abstract

- $\underline{\operatorname{PDF}(1245 \mathrm{~K})}$ 
- $\underline{\text { References }}$

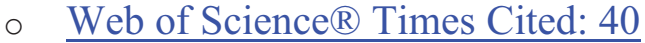

- Go here for SFX

- Elphic, R. C., D. J. Lawrence, W. C. Feldman, B. L. Barraclough, S. Maurice, A. B. Binder, and P. G. Lucey (1998), Lunar Fe and Ti abundances: Comparison of Lunar Prospector and Clementine data, Science, 281, 1493-1496.

- CrossRef,

- CAS,

- $\quad$ Web of Science ${ }^{\circledR}$ Times Cited: 51 ,

- $\underline{\text { ADS }}$

- Go here for SFX

- Elphic, R. C., D. J. Lawrence, W. C. Feldman, B. L. Barraclough, S. Maurice, A. B. Binder, and P. G. Lucey (2000), Determination of lunar global rare earth element abundances using Lunar Prospector neutron spectrometer observations, J. Geophys. Res., 105(E8), 20,333-20,345, doi:10.1029/1999JE001176.

Direct Link:
- $\underline{\text { Abstract }}$
- $\underline{\operatorname{PDF}(1989 \mathrm{~K})}$
- References
- Go here for SFX

- Elphic, R. C., D. J. Lawrence, W. C. Feldman, B. L. Barraclough, O. M. Gasnault, S. Maurice, P. G. Lucey, D. T. Blewett, and A. B. Binder (2002), Lunar Prospector neutron spectrometer constraints on $\mathrm{TiO}_{2}$, J. Geophys. Res., 107(E4), 5024, doi: $10.1029 / 2000 \mathrm{JE} 001460$.

Direct Link:
- Abstract
- Full Article (HTML)
- $\underline{\operatorname{PDF}(3161 \mathrm{~K})}$
- References
- Web of Science ${ }^{\circledR}$ Times Cited: 28
- Go here for SFX

- Feldman, W. C., and D. M. Drake (1986), A Doppler filter technique to measure the hydrogen content of planetary surfaces, Nucl. Instrum. Methods Phys. Res., Sect. A, 245, 182-190, doi:10.1016/0168-9002(86)90274-3.

- CrossRef,

- Web of Science ${ }^{\circledR}$ Times Cited: 34 ,

- $\mathrm{ADS}$

- $\underline{\text { Go here for SFX }}$

- Feldman, W. C., B. L. Barraclough, S. Maurice, R. C. Elphic, D. J. Lawrence, D. R. Thomsen, and A. B. Binder (1998a), Major compositional units of the Moon: Lunar Prospector thermal and fast neutrons, Science, 281, 1489-1493, doi: $10.1126 /$ science.281.5382.1489. 
- CrossRef,

- CAS,

- Web of Science ${ }^{\circledR}$ Times Cited: 51,

- $\underline{\mathrm{ADS}}$

- Go here for SFX

- Feldman, W. C., S. Maurice, A. B. Binder, B. L. Barraclough, R. C. Elphic, and D. J. Lawrence (1998b), Fluxes of fast and epithermal neutrons from Lunar Prospector: Evidence for water ice at the lunar poles, Science, 281, 1496-1500, doi:10.1126/science.281.5382.1496.

- CrossRef,

- PubMed,

- CAS,

- Web of Science® Times Cited: 203,

- ADS

- Go here for SFX

- Feldman, W. C., D. J. Lawrence, R. C. Elphic, B. L. Barraclough, S. Maurice, I. Genetay, and A. B. Binder (2000), Polar hydrogen deposits on the Moon, J. Geophys. Res., 105(E2), 4175-4195, doi:10.1029/1999JE001129.

Direct Link:

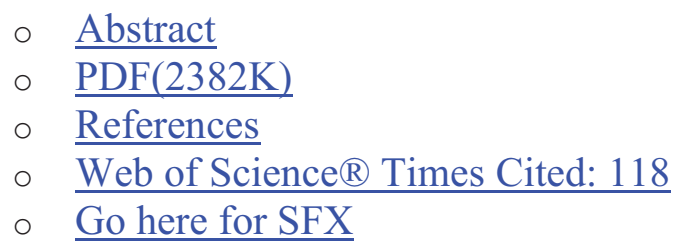

- Feldman, W. C., et al. (2001), Evidence for water ice near the lunar poles, J. Geophys. Res., 106(E10), 23,231-23,251, doi:10.1029/2000JE001444.

Direct Link:

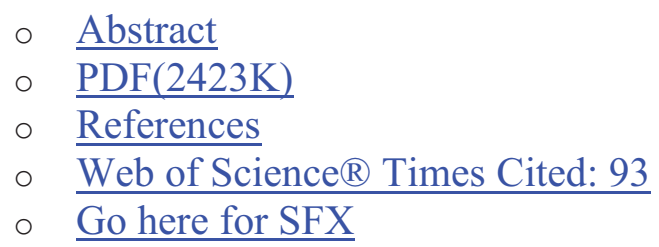

- Feldman, W. C., et al. (2004), Gamma-ray, neutron, and alpha-particle spectrometers for the Lunar Prospector mission, J. Geophys. Res., 109, E07S06, doi:10.1029/2003JE002207.

Direct Link:
- Abstract
- Full Article (HTML)
- PDF(692K)
- References 
- $\underline{\mathrm{ADS}}$

- Go here for SFX

- Little, R. C., et al. (2003), Latitude variation of the subsurface lunar temperature: Lunar Prospector thermal neutrons, J. Geophys. Res., 108(E5), 5046,

doi:10.1029/2001JE001497.

Direct Link:

- Abstract

- Full Article (HTML)

- $\operatorname{PDF}(697 \mathrm{~K})$

- References

- Web of Science $\mathbb{R}$ Times Cited: 4

- Go here for SFX

- Litvak, M. L., I. G. Mitrofanov, A. S. Kozyrev, A. B. Sanin, V. I. Tretyakov, W. V. Boynton, N. J. Kelly, D. Hamara, C. Shinohara, and R. S. Saunders (2006), Comparison between polar regions of Mars from HEND/Odyssey data, Icarus, 180(1), 23-37, doi:10.1016/j.icarus.2005.08.009.

- CrossRef,

- Web of Science ${ }^{\circledR}$ Times Cited: 23,

- $\underline{\text { ADS }}$

- Go here for SFX

- Maurice, S., W. C. Feldman, D. J. Lawrence, O. Gasnault, C. d'Uston, and P. G. Lucey (2000), High-energy neutrons from the Moon, J. Geophys. Res., 105(E8), 20,365-20,375, doi:10.1029/1999JE001151.

Direct Link:

- $\underline{\text { Abstract }}$

- $\underline{\text { PDF }(1215 \mathrm{~K})}$

- References

- Web of Science ${ }^{\circledR}$ Times Cited: 28

- Go here for SFX

- Maurice, S., D. J. Lawrence, W. C. Feldman, R. C. Elphic, and O. Gasnault (2004), Reduction of neutron data from Lunar Prospector, J. Geophys. Res., 109, E07S04, doi: $10.1029 / 2003 J E 002208$.

Direct Link:
- $\quad$ Abstract
- Full Article (HTML)
- $\underline{\operatorname{PDF}(2945 \mathrm{~K})}$
- References

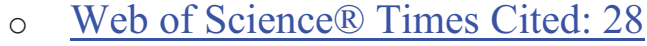
- Go here for SFX 
- Metzger, A. E., J. I. Trombka, L. E. Peterson, R. C. Reedy, and J. R. Arnold (1973), Lunar surface radioactivity: Preliminary results of the Apollo 15 and Apollo 16 gamma ray spectrometer experiments, Science, 179, 800-803, doi:10.1126/science.179.4075.800.

- CrossRef,

- $\underline{\text { CAS }}$,

- Web of Science® Times Cited: 82,

- ADS

- Go here for SFX

- Mitrofanov, I. G., et al. (2008), Experiment LEND of the NASA Lunar Reconnaissance Orbiter for High-Resolution Mapping of Neutron Emission of the Moon, Astrobiology, 8(4), 793-804, doi:10.1089/ast.2007.0158.

- CrossRef,

- PubMed,

- CAS,

- Web of Science ${ }^{\circledR}$ Times Cited: 14,

- $\underline{\text { ADS }}$

- Go here for SFX

- Mitrofanov, I. G., M. L. Litvak, V. I. Tretyakov, M. I. Mokrousov, A. V. Malakhov, and A. Vostrukhin (2009), Neutron components of radiation environment in the near-Earth and near-Mars space, Planet. Space Sci., 57, 1993-1995.

- CrossRef,

- $\underline{\text { CAS }}$,

- Web of Science ${ }^{\circledR}$ Times Cited: 1 ,

- ADS

- Go here for SFX

- Mitrofanov, I. G., et al. (2010a), Lunar Exploration Neutron Detector for the NASA Lunar Reconnaissance Orbiter, Space Sci. Rev., 150(1-4), 183-207, doi:10.1007/s11214-009-9608-4.

- CrossRef,

- Web of Science® Times Cited: 20,

- ADS

- Go here for SFX

- Mitrofanov, I. G., et al. (2010b), Hydrogen mapping of the lunar south pole using the LRO Neutron Detector Experiment LEND, Science, 330, 483-486.

- CrossRef,

- CAS,

- Web of Science ${ }^{\circledR}$ Times Cited: 37,

- $\underline{\text { ADS }}$

- Go here for SFX

- Mitrofanov, I. G., W. V. Boynton, M. L. Litvak, A. B. Sanin, and R. D. Starr (2011), Response to comment on "Hydrogen mapping of the lunar south pole using the LRO Neutron Detector Experiment LEND, " Science, 334, 1058, doi:0.1126/science.1203483.

- CrossRef,

- CAS,

- Web of Science® Times Cited: 5 , 
$\circ \quad \underline{A D S}$

- Go here for SFX

- Press, W. H., S. A. Teukolsky, W. T. Vetterling, and B. P. Flannery (1992), Numerical Recipes in C, Cambridge Univ. Press, Cambridge, U. K.

- Go here for SFX

- Sanin, A. B., et al. (2012), Testing lunar permanently shadowed regions for water ice: LEND Results from LRO, J. Geophys. Res., doi:10.1029/2011JE003971, in press.

Direct Link:

- Abstract

- Full Article (HTML)

- PDF(1714K)

- References

- Web of Science ${ }^{\circledR}$ Times Cited: 3

- Go here for SFX

- Vinogradov, A. P., Y. A. Surkov, G. N. Chernov, F. F. Kirnozov, and G. B. Nazarkina (1966), Measurement of the gamma radiation of the lunar surface by the Luna-10 spacecraft, Cosmic Res., Engl. Transl., 4, 751-755.

- $\underline{\mathrm{ADS}}$

- Go here for SFX

\section{Supporting Information}

\section{Filename Format Size Description}

jgre3010-sup-0001-t01.txt plain text document 3K Tab-delimited Table 1.

Please note: Wiley-Blackwell is not responsible for the content or functionality of any supporting information supplied by the authors. Any queries (other than missing content) should be directed to the corresponding author for the article. 